\title{
The Construction of Orthonormal Wavelets Using Symbolic Methods and a Matrix Analytical Approach for Wavelets on the Interval
}

\author{
Frédéric Chyzak, Peter Paule, Otmar Scherzer, Armin Schoisswohl, and
}

Burkhard Zimmermann

\section{CONTENTS}

1. Introduction

2. Orthonormal Wavelets on $\mathbb{R}$

3. Closed Form Representation of Filter Coefficients

4. Wavelets on the Interval

5. Explicit Results

Acknowledgments

References

Chyzak and Paule were partially supported by the SFB grant F1305 of the Austrian Science Foundation. Scherzer was partially supported by the SFB grant F1310 of the Austrian Science Foundation. Schoisswohl was partially supported by the Upper Austrian Government. At the time of submission Scherzer and Schoisswohl were at the Industrial Mathematics Institute, Johannes Kepler University, Linz, Austria.
We discuss closed form representations of filter coefficients of wavelets on the real line, half real line and on compact intervals. We show that computer algebra can be applied to perform this task. Moreover, we present a matrix analytical approach that unifies constructions of wavelets on the interval.

\section{INTRODUCTION}

Wavelets are one of the most popular tools in signal and image processing. These functions are widely used in many practical applications such as data compression [Bradley et al. 1993; DeVore et al. 1992; Scherzer et al. 1998], or for the solution of partial differential equations (see [Jaffard 1992], for example). Wavelets are special functions that often have a fractal character. This makes it relatively difficult to work with them explicitly; for example, point evaluation of a wavelet function may already be a computationally expensive task. To work with wavelets one uses the nice feature that they are defined by a small number of parameters, called filter coefficients. In general, any algorithm relying on wavelets uses the filter coefficients only and not the wavelet function itself.

In this paper we review the basic equations for the filter coefficients. We show that these equations can be solved using computer algebra. In particular we can construct closed form representations of the wavelet coefficients (Section 3). The most popular wavelets form an orthonormal basis of the space of square integrable functions on $\mathbb{R}$ [Daubechies 1992]. In many practical applications one requires a basis on the half-line or on a compact interval. In Sec- 
tion 4 we review several constructions of wavelets on the interval. We construct the filter coefficients of wavelets on the interval using a matrix analytical approach which has the advantage to unify several constructions in the literature [Meyer 1991; Cohen et al. 1993b; Dahmen et al. 1999]. Moreover, our construction reveals that there exists a closed form representation of the filter coefficients of wavelets on the interval. Another big advantage of using computer algebra is that one can avoid instabilities which occur in numerical calculations of filter coefficients.

Sections $4 \mathrm{E}$ and 5 summarize the algorithms for calculating closed form coefficients of wavelets on the real line and on compact intervals and present some results.

\section{ORTHONORMAL WAVELETS ON $\mathbb{R}$}

For convenience we summarize here the well-known construction of compactly supported orthonormal wavelet bases for $L^{2}(\mathbb{R})$ (for details, see [Daubechies 1988; 1992; 1993; Cohen and Daubechies 1993], for example).

The construction of wavelets is related with the construction of a scaling function $\varphi$ such that for fixed $m \in \mathbb{Z}$ the functions $\varphi_{m, k}:=2^{-m / 2} \varphi\left(2^{-m} x-k\right)$, with $k \in \mathbb{Z}$, are orthonormal with respect to $L^{2}(\mathbb{R})$. Moreover, the spaces

$$
V_{m}:=\overline{\operatorname{span}\left\{\varphi_{m, k}: k \in \mathbb{Z}\right\}}
$$

constitute a multiresolution analysis for $L^{2}(\mathbb{R})$, i.e.,

$$
V_{m} \subset V_{m-1}, \quad \text { for } m \in \mathbb{Z},
$$

with

$$
\bigcap_{m \in \mathbb{Z}} V_{m}=\{0\} \quad \text { and } \overline{\bigcup_{m \in \mathbb{Z}} V_{m}}=L^{2}(\mathbb{R}) .
$$

The wavelet spaces $W_{m}$ are the orthogonal complements of $V_{m}$ in $V_{m-1}$, i.e.,

$$
W_{m}:=V_{m}^{\perp} \cap V_{m-1} .
$$

One defines the wavelet $\psi$ such that the functions $\psi_{m, k}:=2^{-m / 2} \psi\left(2^{-m} x-k\right), \quad k \in \mathbb{Z}$, form an orthonormal basis for $W_{m}$. Since both $V_{m}$ and $W_{m}$ are contained in $V_{m-1}$ the scaling function $\varphi$ must satisfy the dilation equation

$$
\varphi(x)=\sum_{k \in \mathbb{Z}} h_{k} \varphi(2 x-k),
$$

where the sequence $\left\{h_{k}\right\}$ is known as the filter sequence and satisfies constraints to be recalled below. Correspondingly, the wavelet $\psi$ satisfies

$$
\psi(x)=\sum_{k \in \mathbb{Z}} g_{k} \varphi(2 x-k),
$$

where $g_{k}=(-1)^{k} h_{1-k}$.

Daubechies [1988] established conditions on the filter sequence in order to ensure that the dilation equation $(2-1)$ has a solution $\varphi \in L^{2}(\mathbb{R})$, with

$$
\operatorname{supp} \varphi=[1-N, N]
$$

for a given integer $N$, and that for fixed $m$ the functions $\varphi_{m, k}$ are orthogonal with the property that polynomials up to degree $N-1$ can be represented as linear combinations of $\varphi_{m, k}$. Compact support of $\varphi$ in $[-N+1, N]$ is ensured by

$$
h_{k}=0, \text { for } k<1-N \text { or } k>N .
$$

A requirement for the existence of a solution of $(2-1)$ is

$$
\sum_{k=1-N}^{N} h_{k}=2
$$

which is equivalent to $\int \varphi(x) d x=1$. Orthonormality of the translates of $\varphi$, i.e., the condition that $\int \varphi(x) \varphi(x-l) d x=\delta_{0, l}$, can be translated into

$$
\sum_{k=1-N}^{N} h_{k} h_{k-2 l}=2 \delta_{0, l}, \text { for } l=0, \ldots, N-1,
$$

and the condition that polynomials be representable by the $\varphi_{m, k}$ leads to $\int x^{l} \psi(x) d x=0$ for $l=0, \ldots$, $N-1$, which is equivalent to

$$
\sum_{k=1-N}^{N}(-1)^{k} h_{1-k} k^{l}=0, \text { for } l=0, \ldots, N-1 .
$$

\section{CLOSED FORM REPRESENTATION OF FILTER COEFFICIENTS}

In this section we reconsider the calculation of the filter coefficients $h_{k}$ from equations $(2-3)-(2-6)$ by using methods of computer algebra. Below we give a brief and informal account on Gröbner bases, which we exemplify by the calculation of the filter coefficients for the special case $N=2$. Afterwards we pass on to the cases $N>2$ and present more computational details on our symbolic approach. 


\section{A. Gröbner Bases}

Due to the conditions imposed explicitly on the summation bounds in the equations $(2-4)-(2-6)$, we can restrict our attention to the task of solving only those; the conditions (2-3) can be satisfied separately by mere definition. But instead of solving the equations (2-4)-(2-6) numerically and for a fixed integer value $N$, we try to find closed forms for the coefficients $h_{k}$, i.e., to approach the problem from the symbolic computation point of view. To solve systems of polynomial equations symbolically, the obvious tools to use are Gröbner bases: after their original introduction by B. Buchberger [1965] to answer ideal-theoretic questions, the solving of algebraic systems was soon realized to be one of their natural domains of application [Buchberger 1970]. For further introductory information see, e.g., [Winkler 1996] or [von zur Gathen and Gerhard 1999]. Additional references and the state of art can be found in [Buchberger and Winkler 1998].

The case $N=1$ is trivial; $h_{0}=h_{1}=1$ is the only solution. Hence we illustrate the Gröbner bases method for $N=2$. In this case we are interested in all common roots of the five polynomials in the four variables $x_{1}, x_{2}, x_{3}, x_{4}$ :

$$
\begin{aligned}
& -2+x_{1}+x_{2}+x_{3}+x_{4},-2+x_{1}^{2}+x_{2}^{2}+x_{3}^{2}+x_{4}^{2}, \\
& x_{1} x_{3}+x_{2} x_{4}, x_{1}-x_{2}+x_{3}-x_{4}, \quad 2 x_{1}-x_{2}+x_{4} ;
\end{aligned}
$$

for the sake of simplicity we introduced the following renaming of variables:

$$
x_{1}=h_{-1}, x_{2}=h_{0}, x_{3}=h_{1} \text {, and } x_{4}=h_{2} .
$$

Let $I$ be the ideal in the polynomial ring

$$
\mathbb{C}\left[x_{1}, x_{2}, x_{3}, x_{4}\right]
$$

generated by the polynomials from (3-1). Applying Buchberger's algorithm with respect to a certain order imposed on the monomials of $\mathbb{C}\left[x_{1}, x_{2}, x_{3}, x_{4}\right]$ (here: "lexicographic with $x_{4}>x_{3}>x_{2}>x_{1}$ ") delivers an alternative description of the ideal $I$, namely by the generators:

$$
\begin{array}{cc}
-1-4 x_{1}+8 x_{1}^{2}, & -1-2 x_{1}+2 x_{2}, \\
-1+x_{1}+x_{3}, & -1+2 x_{1}+2 x_{4} .
\end{array}
$$

The polynomials (3-2) again generate the ideal $I$, and in particular, share the same variety of common roots as the generators from (3-1). But additionally, they form a Gröbner basis of $I$. Due to the choice of a lexicographic monomial order, they furthermore possess the following "elimination property": the first polynomial in the Gröbner basis is a univariate polynomial (here in $x_{1}$ ), the second one a bivariate polynomial that involves only one further variable (here $x_{1}$ and $x_{2}$ ), the third one a polynomial in three variables (here $x_{1}, x_{2}$, and $x_{3}$ ), and so on. In other words, the role of the Gröbner basis algorithm in solving systems of algebraic equations is the same as that of Gaussian elimination in solving systems of linear equations, namely to triangularize the system or to carry out the elimination, respectively.

Remarkably, in our situation of solving filter coefficient equations, an even nicer pattern emerges. Indeed, given the first univariate Gröbner basis polynomial $p_{1}\left(x_{1}\right)$ in $x_{1}$ only, the second Gröbner basis polynomial is the sum of a univariate polynomial in $x_{1}$ and a linear polynomial in $x_{2}$; the third Gröbner basis polynomial is the sum of a univariate polynomial in $x_{1}$ and a linear polynomial in $x_{3}$, and so on. This means that all other filter coefficients $x_{i}$ for $i>1$ find a representation of the form

$$
x_{i}=p_{i}\left(x_{1}\right),
$$

where each $p_{i}\left(x_{1}\right)$ is a polynomial from $\mathbb{C}\left[x_{1}\right]$, i.e., depending on $x_{1}$ only. Consequently, there are as many different solutions of a system of filter coefficient equations as there are different roots of the first univariate Gröbner basis polynomial $p_{1}\left(x_{1}\right)$. So far we have observed the nice pattern of a univariate polynomial and relations like (3-3) for all values of $N$ up to 6 , so that we conjecture that this situation also holds for arbitrary $N$. For readers interested in ideal theory we state this in the form of the following conjecture. (For more ideal-theoretic background see, for instance, the "shape lemma" in [Winkler 1996].)

Conjecture 3.1. Polynomial ideals corresponding to Daubechies filter coefficient equations are 0-dimensional and radical.

Also note that by choosing different lexicographic orders on the $x_{i}$, it would be possible to obtain alternative descriptions of the solutions, parameterized by another choice of $x_{j}$. Also, since we expect a representation of the form of $(3-3)$, other nonlexicographic orders could be used, with enhanced efficiency. (Specifically, any order which sorts $x_{2}$, 
$x_{3}$, and $x_{4}$ by any non-lexicographic order, but sorts them lexicographically higher than $x_{1}$.) In the same vein, extensive calculations discussed elsewhere have shown that for $N=2, \ldots, 6$, the ideal generated by the system $(2-4)-(2-6)$ contains a nonzero univariate polynomial in $h_{i}$ of degree $2^{N-1}$ for each $i$ between $1-N$ and $N$, and none of smaller degree. (See related explicit results in Table 2 on page 83 and the surrounding text.)

To conclude this informal discussion of the Gröbner bases approach, we state the solution of the case $N=2$ explicitly. Since $(1+\sqrt{3}) / 4$ and $(1-\sqrt{3}) / 4$ are the roots of the first Gröbner basis polynomial $-1-4 x_{1}+8 x_{1}^{2}$, we obtain two solutions for the filter coefficients:

$$
\begin{aligned}
& \left(x_{1}, x_{2}, x_{3}, x_{4}\right)=\frac{1}{4}(1+\sqrt{3}, 3+\sqrt{3}, 3-\sqrt{3}, 1-\sqrt{3}), \\
& \left(x_{1}, x_{2}, x_{3}, x_{4}\right)=\frac{1}{4}(1-\sqrt{3}, 3-\sqrt{3}, 3+\sqrt{3}, 1+\sqrt{3}) .
\end{aligned}
$$

\section{B. Reduction of the Filter Coefficient Equations}

For fixed $N$, the system $(2-4)-(2-6)$ consists of $2 N+$ 1 equations in $2 N$ unknowns. For each $N$ such that the system admits a nice triangular representation of the form (3-3), $2 N$ is also the number of Gröbner basis polynomials we finally have to solve explicitly. In this section, as an important preprocessing step to Gröbner basis computation, we transform the system (2-4)-(2-6) into a more economic form. More precisely, this system will consist of only $N$ equations in $N$ unknowns; the corresponding Gröbner bases will then consist of $N$ polynomials for which one again observes the nice shape that was described above (see the discussion preceding Conjecture 3.1). Not only is this system more compact, but it also induce faster Gröbner basis calculations in practice, as was suggested by the doubly exponential upper bound of the degree of polynomials in a Gröbner basis in terms of the number of variables.

In a first reduction step we introduce a normalization via multiplication by a binomial coefficient; namely, for any fixed positive integer $N$ we define $a_{k}$ by

$$
h_{k}=\left(\begin{array}{c}
2 N-1 \\
N-k
\end{array}\right) a_{N-k}, \quad(k=1-N, \ldots, N) .
$$

This implicitly installs conditions (2-3) and thus enables to relax the explicit statement of the summation bounds in $(2-4)-(2-6)$.
More importantly, a second change of variables will prove successful in the sequel: for fixed positive integer $N$ we restrict ourselves to consider $a_{k}$ as a polynomial in $k$ of degree at most $N-1$. To this end, we write

$$
a_{k}=\sum_{j=0}^{N-1} P_{j}\left(\begin{array}{l}
k \\
j
\end{array}\right),
$$

where the $P_{j}$ are the new unknowns we have to solve for. Note that we have in total $N$ of those, instead of $2 N$ in the original setting $(2-4)-(2-6)$. In addition, we shall see below why it is convenient to work with the $\left(\begin{array}{c}k \\ j\end{array}\right)$ as basis elements instead of the $k^{j}$.

With ansatz (3-4) and (3-5) in hand, we return to equations $(2-3)-(2-6)$. It is not difficult to see that only two of them remain: $(2-3)$ is guaranteed due to the presence of the binomial coefficient in (3-4); also, equation (2-6) is satisfied for arbitrary $l=0, \ldots, N-1$ because of the following elementary combinatorial lemma (see [Graham et al. 1994, (5.42)], for instance).

Lemma 3.2. For any nonnegative integer $n$ and complex numbers $c_{i}$ :

$\sum_{k=0}^{n}(-1)^{k}\left(\begin{array}{l}n \\ k\end{array}\right)\left(c_{0}+c_{1} k+\cdots+c_{n} k^{n}\right)=(-1)^{n} n ! c_{n}$.

Now, with ansatz $(3-4)$, equation $(2-6)$ for $0 \leq l \leq$ $N-1$ is rewritten as

$$
\sum_{k=0}^{2 N-1}(-1)^{k-N+1}\left(\begin{array}{c}
2 N-1 \\
k
\end{array}\right) a_{k}(k-N+1)^{l}=0,
$$

and both $a_{k}$ and $(k-N+1)^{l}$ are polynomials in $k$ with degree less than or equal to $N-1$. Hence, by Lemma 3.2 , equation $(2-6)$ is satisfied for all $l$ in question.

In order to state new versions of the remaining equations $(2-4)$ and $(2-5)$ in the form of propositions, it is convenient to renormalize $P_{j}$ by introducing

$$
Q_{j}=\left(\begin{array}{c}
2 N-1 \\
j
\end{array}\right) P_{j}
$$

The final ansatz now becomes

$$
h_{k}=\sum_{j=0}^{N-1} Q_{j}\left(\begin{array}{c}
2 N-j-1 \\
N+k-1
\end{array}\right)
$$


after substituting (3-5) into (3-4) and using the elementary fact

$$
\left(\begin{array}{c}
2 N-1 \\
k
\end{array}\right)\left(\begin{array}{c}
k \\
j
\end{array}\right)=\left(\begin{array}{c}
2 N-1 \\
j
\end{array}\right)\left(\begin{array}{c}
2 N-j-1 \\
2 N-k-1
\end{array}\right)
$$

Proposition 3.3. Under the assumption (3-7), equation (2-4) is equivalent to

$$
\sum_{j=0}^{N-1} 2^{2 N-j-2} Q_{j}=1
$$

Proof. This follows from

$$
\sum_{l}\left(\begin{array}{c}
2 N-j-1 \\
l
\end{array}\right)=2^{2 N-j-1}
$$

a special instance of the binomial theorem.

Proposition 3.4. Under the assumption (3-7), equation $(2-5)$ is equivalent to

$$
\sum_{i, j=0}^{N-1}\left(\begin{array}{c}
4 N-i-j-2 \\
2 N+2 l-i-1
\end{array}\right) Q_{i} Q_{j}=2 \delta_{0, l}
$$

for $l=0, \ldots, N-1$.

Proof. Substituting (3-7) twice in $(2-5)$ yields

$$
\sum_{i, j=0}^{N-1} Q_{i} Q_{j} \sum_{k=1-N}^{N}\left(\begin{array}{c}
2 N-j-1 \\
N+k-1
\end{array}\right)\left(\begin{array}{c}
2 N-i-1 \\
N+k-2 l-1
\end{array}\right)=2 \delta_{0, l} .
$$

The inner sum can be evaluated as follows: after changing $k$ into $N-k$ and applying the binomial symmetry

$$
\left(\begin{array}{c}
n \\
m
\end{array}\right)=\left(\begin{array}{c}
n \\
n-m
\end{array}\right)
$$

to the second binomial, it becomes

$$
\sum_{k=0}^{2 N-1}\left(\begin{array}{c}
2 N-j-1 \\
2 N-k-1
\end{array}\right)\left(\begin{array}{c}
2 N-i-1 \\
k+2 l-i
\end{array}\right)=\left(\begin{array}{c}
4 N-i-j-2 \\
2 N+2 l-i-1
\end{array}\right)
$$

where the last identity is a variant of the standard Vandermonde summation; see [Graham et al. 1994, (5.22)], for instance. The preceding derivation is clearly invertible.

The number of equations can be reduced further. In order to prove this, we need another elementary combinatorial result.

Lemma 3.5. For non-negative integers $m$ and $n$ such that $m+n \geq 1$ :

$$
\sum_{l}\left(\begin{array}{c}
m+n \\
m+2 l
\end{array}\right)=2^{m+n-1}
$$

Proof. We have

$$
\sum_{l}\left(\begin{array}{c}
m+n \\
l
\end{array}\right)=2^{m+n} \quad \text { and } \quad \sum_{l}(-1)^{l}\left(\begin{array}{c}
m+n \\
l
\end{array}\right)=0
$$

as a result of the binomial theorem. Taking the sum and the difference of both identities yields

$$
\sum_{l}\left(\begin{array}{c}
m+n \\
2 l
\end{array}\right)=\sum_{l}\left(\begin{array}{c}
m+n \\
2 l+1
\end{array}\right)=2^{m+n-1} .
$$

Now, the sum in the claim is one of the two sums above, depending on the parity of $m$, whence the result.

Remark. Proofs of binomial summations like the Vandermonde formula or Lemma 3.5 can now be carried out in a purely automatic fashion thanks to Zeilberger's summation machinery [Petkovšek et al. 1996]; see, for instance, the Mathematica package [Paule and Schorn 1995].

We are now ready to carry out the last reduction step. As opposed to Propositions 3.3 and 3.4 that relate identities between the $h_{i}$ and the $Q_{i}$, the following proposition states an reduction between the equations in the $Q_{i}$ only.

Proposition 3.6. Under the simultaneous assumption of the cases $l=1, \ldots, N-1$ in (3-9), the case $l=0$ in equation (3-9) is equivalent to

$$
\left(\sum_{j=0}^{N-1} 2^{2 N-j-2} Q_{j}\right)^{2}=1
$$

and is therefore a consequence of equation (3-8).

Proof. By $L_{l}$ we denote the double sum on the lefthand side of $(3-9)$. We assume the cases $l=1, \ldots$, $N-1$ in (3-9). Because of the symmetry property $L_{l}=L_{-l}$ and after applying Lemma 3.5, we have

$$
\begin{aligned}
L_{0} & =\sum_{l=1-N}^{N-1} L_{l}=\sum_{i, j=0}^{N-1} 2^{4 N-i-j-3} Q_{i} Q_{j} \\
& =2\left(2^{2(N-1)} \sum_{j=0}^{N-1} \frac{Q_{j}}{2^{j}}\right)^{2} .
\end{aligned}
$$

This proves that the relation $L_{0}=2$ is equivalent to equation $(3-10)$.

Finally we summarize our reduction of the $2 N+1$ Daubechies equations in $2 N$ unknowns to $N$ algebraic equations in $N$ unknowns as follows: 
Proposition 3.7. Any solution of the $N$ algebraic equations

and

$$
\sum_{j=0}^{N-1} \frac{Q_{j}}{2^{j}}=\frac{1}{2^{2 N-2}},
$$

$\sum_{i, j=0}^{N-1}\left(\begin{array}{c}4 N-i-j-2 \\ 2 N+2 l-i-1\end{array}\right) Q_{i} Q_{j}=0$,

$$
\text { for } l=1, \ldots, N-1 \text {, }
$$

gives rise to a solution of the Daubechies filter coefficient equations (2-3)-(2-6) via

$h_{k}=\sum_{j=0}^{N-1}\left(\begin{array}{c}2 N-j-1 \\ N+k-1\end{array}\right) Q_{j}$,

$$
\text { for } k=1-N, \ldots, N \text {. }
$$

Conversely for any solution $h$ of $(2-3)-(2-6)$, any solution $Q$ of (3-13) is a solution of (3-11)-(3-12).

At this stage, the first part of the proposition provides us with a means to obtain solutions of (2-3)$(2-6)$. But this procedure may miss solutions $h$ : for $h$ satisfying (2-3)-(2-6), we have no proof yet that the system (3-13) is solvable as a system in $Q$. But this gap can also be closed, leading to the following more specific "Equivalence Theorem," whose proof is postponed to the next section.

Theorem 3.8 (Equivalence Theorem). Systems (2-3)(2-6) and (3-11)-(3-12) are equivalent descriptions of the same algebraic variety. More precisely, there exists an explicit linear isomorphism between $\mathbb{C}^{N}$ and the $N$-dimensional linear subspace defined by $(2-6)$ in $\mathbb{C}^{2 N}$ which realizes a linear change of coordinates between the solution set of the algebraic system (2-4)-(2-6) and the solution set of the algebraic system $(3-11)-(3-12)$.

In particular, this theorem provides a bijection between the solution sets of both systems; additionally the whole structures of these solution sets, including dimension and multiplicities, are the same.

Remark. In connection with experimental mathematics it is worth noting that concrete Gröbner bases computations led us to conjecture the Equivalence Theorem. Namely, it turned out that the Gröbner bases computed with respect to the system (3-11)$(3-12)$ have the same nice triangulation property as those computed with respect to the system $(2-4)-$ $(2-6)$. In addition we observed that in all instances the first univariate Gröbner basis polynomial is the same in both cases.

\section{C. Proof of the Equivalence Theorem}

This section is devoted to the proof of Theorem 3.8. Viewing (2-4)-(2-5) as defining an algebraic variety $\mathcal{H}$ in $\mathbb{C}^{2 N}$ and $(3-11)-(3-12)$ as defining an algebraic variety $Q$ in $\mathbb{C}^{N}$, we are about to show that (3-13) installs a linear isomorphism between the $N$-dimensional linear subspace $\mathcal{K}$ defined by $(2-6)$ in $\mathbb{C}^{2 N}$ and $\mathbb{C}^{N}$. In this way $\mathcal{H} \cap \mathcal{K}$ and $\mathcal{Q}$ can be viewed as the same algebraic variety in $\mathbb{C}^{N} \simeq \mathcal{K}$, but expressed in different linear bases.

The proof consists of three steps: first, we introduce an injective linear map $\alpha$ from $\mathbb{C}^{N}$ to $\mathbb{C}^{2 N}$, which embeds $\mathcal{Q}$ into $\mathcal{H}$; next, we introduce a surjective linear map $\beta$ from $\mathbb{C}^{2 N}$ to $\mathbb{C}^{N}$, whose kernel is $\mathcal{K}$; finally, by showing $\beta \alpha=0$, we obtain the isomorphism between the algebraic varieties $Q$ and $\mathcal{H} \cap \mathcal{K}$.

The Injective Linear Map $\alpha$. Following the idea of embedding $Q$ into $\mathcal{H}$, we introduce the mapping

$$
\alpha: \mathbb{C}^{N} \rightarrow \mathbb{C}^{2 N}
$$

which maps $Q=\left(Q_{0}, \ldots, Q_{N-1}\right)^{t}$ to the point $h=$ $\left(h_{1-N}, \ldots, h_{N}\right)^{t}$ given by

$$
h_{i}=\sum_{j=0}^{N-1}\left(\begin{array}{c}
2 N-j-1 \\
N+i-1
\end{array}\right) Q_{j} .
$$

Denote by $\alpha=\left(\alpha_{i, j}\right)$ the matrix of this linear map in the canonical bases of $\mathbb{C}^{N}$ and $\mathbb{C}^{2 N}$, where the indices $i$ and $j$ range from 1 to $2 N$, and 1 to $N$, respectively. In view of the unusual indexing in $h$ and $Q$, we have

$$
h_{i-N}=\sum_{j=1}^{N} \alpha_{i, j} Q_{j-1},
$$

and by identification

$$
\alpha_{i, j}=\left(\begin{array}{c}
2 N-j \\
i-1
\end{array}\right)
$$

for $1 \leq i \leq 2 N$ and $1 \leq j \leq N$. Therefore,

$$
\alpha=\left(\begin{array}{c}
S \\
U
\end{array}\right)
$$

for two $N \times N$ square blocks $S$ and $U$. The antidiagonal of $U$ is obtained when $i-N=N+1-j$, 
i.e., $i-1=2 N-j$, for which $\alpha_{i, j}=1$; the triangular block under the antidiagonal of $U$ is obtained when $i-N>N+1-j$, i.e., $i-1>2 N-j$, for which $\alpha_{i, j}=0$; the block $U$ is therefore upper antitriangular with 1 's on the antidiagonal. It follows that $\alpha$ has maximal rank, namely $N$. By the rank theorem we obtain that $\operatorname{dim} \operatorname{ker} \alpha=0$ and thus that $\alpha$ is injective.

Now consider $Q \in \mathcal{Q}$. In other words, $Q$ satisfies (3-11)-(3-12), so that by the first part of Proposition 3.7, equations $(2-4)-(2-6)$ are satisfied by $h=$ $\alpha Q$. In particular, equations $(2-4)-(2-5)$ are satisfied, so that $h \in \mathcal{H}$.

Summarizing, the variety $\mathcal{Q}$ defined by $(3-11)-$ $(3-12)$ is injectively mapped by $\alpha$ into the variety $\mathcal{H}$ defined by $(2-4)-(2-5)$ :

$$
\alpha(Q) \subseteq \mathcal{H} .
$$

The Surjective Linear Map $\beta$. Following the idea of regarding the variety defined by $(2-4)-(2-6)$ as an intersection by a suitable kernel $\mathcal{K}$, we introduce the mapping

$$
\beta: \mathbb{C}^{2 N} \rightarrow \mathbb{C}^{N}
$$

which maps $h=\left(h_{1-N}, \ldots, h_{N}\right)^{t}$ to $z=\left(z_{0}, \ldots, z_{N-1}\right)^{t}$ given in analogy with (2-6) by

$$
z_{i}=\sum_{j=1-N}^{N}(-1)^{j} h_{1-j} j^{i} .
$$

Again, we also denote by

$$
\beta=\left(\beta_{i, j}\right)
$$

the matrix of this linear map on the canonical bases of $\mathbb{C}^{2 N}$ and $\mathbb{C}^{N}$, where the indices $i$ and $j$ range from 1 to $N$ and 1 to $2 N$, respectively. In view of the unusual indexing in $z$ and $h$, we have

$$
z_{i-1}=\sum_{j=1}^{2 N} \beta_{i, j} h_{j-N}
$$

and by identification

$$
\beta_{i, j}=(-1)^{N+1-j}(N+1-j)^{i-1}
$$

for $1 \leq i \leq N$ and $1 \leq j \leq 2 N$. Therefore, since the signs only depend on $j$,

$$
\beta=(-1)^{N} \gamma \operatorname{diag}_{2 N}(1,-1, \ldots, 1,-1),
$$

where $\operatorname{diag}_{r}\left(\lambda_{1}, \ldots, \lambda_{r}\right)$ denotes the $r \times r$-diagonal matrix with entries $\lambda_{i}$ on the diagonal, and where $\gamma$ is a matrix

$$
\gamma=\left(\begin{array}{c}
V \\
W
\end{array}\right)
$$

consisting of two $N \times N$ square Vandermonde blocks $V$ and $W$. For example, the first block $V$ is the Vandermonde matrix of the powers of $N$, on the first column, to the powers of 1 , on the last column. It follows from the non-nullity of the Vandermonde determinant that $\beta$ has maximal rank, namely $N$, and is thus surjective.

Now, by the rank theorem the kernel $\mathcal{K}=\operatorname{ker} \beta$ has dimension $N$ and by construction is precisely the linear subspace of $\mathbb{C}^{2 N}$ defined by $(2-6)$. The algebraic variety defined by $(2-4)-(2-6)$ is therefore included in this kernel $\mathcal{K}$; it is $\mathcal{H} \cap \mathcal{K}$, whose study is the topic of the next paragraph.

The Intersection $\mathcal{H} \cap \mathcal{K}$. Since $\alpha$ is injective, it realizes a linear isomorphism between $\mathbb{C}^{N}$ and the image $\alpha\left(\mathbb{C}^{N}\right)$. Proving that this image is the kernel $\mathcal{K}$ of $\beta$ now suffices to obtain that $Q$ is in bijection with the intersection $\mathcal{H} \cap \mathcal{K}$ by this linear isomorphism $\alpha$. This bijection is an isomorphism of algebraic varieties.

To this end, we compute $z=\beta \alpha Q$ for $Q \in \mathbb{C}^{N}$. We have, for $0 \leq l \leq N-1$,

$$
\begin{aligned}
& z_{l}=\sum_{i=1-N}^{N}(-1)^{i} i^{l} h_{1-i}=\sum_{i=0}^{2 N-1}(-1)^{i+1-N}(i+1-N)^{l} h_{N-i} \\
& =\sum_{i=0}^{2 N-1}(-1)^{N+1+i}(i+1-N)^{l} \sum_{j=0}^{N-1}\left(\begin{array}{c}
2 N-j-1 \\
2 N-i-1
\end{array}\right) Q_{j} \\
& =\sum_{j=0}^{N-1}(-1)^{N+1} Q_{j} \sum_{i=0}^{2 N-1}(-1)^{i}\left(\begin{array}{c}
2 N-j-1 \\
i-j
\end{array}\right)(i+1-N)^{l} \\
& =\sum_{j=0}^{N-1}\left((-1)^{N+1+j} Q_{j}\right. \\
& \left.\times \sum_{i=0}^{2 N-1-j}(-1)^{i}\left(\begin{array}{c}
2 N-j-1 \\
i
\end{array}\right)(i+j+1-N)^{l}\right) .
\end{aligned}
$$

We claim that the inner sum is zero, whence $z=$ 0 and $\beta \alpha=0$. Indeed, this is a consequence of Lemma 3.2: the result is obtained when considering identity (3-6) for $n=2 N-j-1$ and polynomials in $i$ (taking the role of $k$ in $(3-6)$ ), and in view of the fact that $(i+j+1-N)^{l}$ is a polynomial in $i$ of 
degree $l$ with $l<n$ since $l \leq N-1$ and $j \leq N-1$, therefore such that $c_{n}=0$.

We know from the analysis of $\alpha$ that $\alpha(\mathcal{Q}) \subseteq \mathcal{H}$, and wish to obtain an equality. We have just obtained $\beta \alpha=0$, so that

$$
\alpha\left(\mathbb{C}^{N}\right) \subseteq \operatorname{ker} \beta=\mathcal{K} .
$$

Now, as already mentioned, by the injectivity of $\alpha$ and the rank theorem applied to $\beta$, the dimensions of $\alpha\left(\mathbb{C}^{N}\right)$ and $\operatorname{ker} \beta$ are both $n$. Thus the equality

$$
\alpha\left(\mathbb{C}^{N}\right)=\operatorname{ker} \beta=\mathcal{K} .
$$

From it and the known inclusion $\alpha(Q) \subseteq \mathcal{H}$ therefore follows

$$
\alpha(\mathcal{Q}) \subseteq \mathcal{H} \cap \mathcal{K}
$$

For the converse inclusion, let $h \in \mathcal{H} \cap \mathcal{K}$. In other words, $h$ satisfies equations $(2-4)-(2-6)$. By the second part of Proposition 3.7, equations (3-11)-(3-12) are satisfied by $Q$, so that $Q \in \mathcal{Q}$. Finally,

$$
\alpha(\mathcal{Q})=\mathcal{H} \cap \mathcal{K} .
$$

Remark. The inverse mapping of $\alpha$ (from $\operatorname{ker} \beta$ to $\mathbb{C}^{N}$ ) is given by

$$
Q_{i}=\sum_{j=1}^{N}(-1)^{N+i+j}\left(\begin{array}{c}
N+j-1 \\
2 N-i-1
\end{array}\right) h_{j}
$$

for $0 \leq i \leq N-1$, as can be obtained from elementary manipulations of binomials and the special case $(1+(-1))^{n}$ of the binomial theorem.

\section{D. Computational Aspects and a Conjecture}

We conclude this section with a few comments on various computational aspects of our approach, together with a conjecture that has been suggested by the observation of the filter coefficients obtained for small values of $N$.

First of all the reduction of the original system to $N$ equations in $N$ unknowns enables the computation of the corresponding Gröbner bases up to $N=$ 6 . We used the computer algebra system Mathematica and the built-in procedure GroebnerBasis (see Section 5). The case $N=6$ takes about 20 seconds and uses less than $10 \mathrm{MB}$ of memory on a computing platform equipped with a Pentium II processor; the case $N=7$ is intractable within $128 \mathrm{MB}$.
Another important point concerns the remark at the end of Section 3B that the Gröbner bases computed with respect to the $N$ equations $(3-11)$ and (3-12) from Proposition 3.7 have the same nice triangulation property as those computed with respect to $(2-4)-(2-6)$. We make this link explicit in the form of a proposition, for which we need the following definition: a polynomial explicit representation (with respect to $x_{1}$ ) of a 0 -dimensional ideal of $\mathbb{C}\left[x_{1}, \ldots, x_{r}\right]$ is a generating system of the ideal of the form

$$
\left\{p_{1}\left(x_{1}\right), x_{2}-p_{2}\left(x_{1}\right), \ldots, x_{r}-p_{r}\left(x_{1}\right)\right\}
$$

for univariate polynomials $p_{i}$. When an ideal has such a representation, the $r$ generators are a Gröbner basis of the ideal for any term order such that $x_{1}<$ $x_{i}$ for $i>1$, and the polynomial $p_{i}$ for $i>1$ can be chosen of smaller degree than $p_{1}$.

Proposition 3.9. Fix an integer $N$. Then, the algebraic system (2-4)-(2-6) admits a polynomial explicit representation with respect to $h_{N}$ if and only if the algebraic system (3-11)-(3-12) admits a polynomial explicit representation with respect to $Q_{0}$. Additionally, the univariate polynomials in both representations are equal (up to a renaming of indeterminates) when this property is satisfied.

Note that in this proposition we consider univariate polynomials in $h_{N}$, instead of univariate polynomials in $h_{1-N}=x_{1}$ as in Section 3A. Explicit values of the common univariate polynomial under consideration are provided for $N$ between 2 and 6 in Table 2 on page 83 .

Proof. For a polynomial explicit representation

$$
\left\{p_{0}\left(h_{N}\right), h_{N-1}-p_{1}\left(h_{N}\right), \ldots, h_{1-N}-p_{2 N-1}\left(h_{N}\right)\right\}
$$

with respect to $h_{N}$ of the ideal generated by the system $(2-4)-(2-6)$, we apply the mapping $\beta$ of Section $3 \mathrm{C}$, which maps a solution $h$ of $(2-4)-(2-6)$ to a solution $Q$ of $(3-11)-(3-12)$. We let $h$ be a solution of $(2-4)-(2-6)$.

First, setting $i=0$ in (3-14) yields $h_{N}=Q_{0}$, so that $p_{0}\left(Q_{0}\right)=p_{0}\left(h_{N}\right)=0$. Next, for any $i>0$, equation $(3-14)$ rewrites

$$
Q_{i}=\sum_{j=1}^{N}(-1)^{N+i+j}\left(\begin{array}{c}
N+j-1 \\
2 N-i-1
\end{array}\right) p_{N-j}\left(h_{N}\right)=q_{i}\left(Q_{0}\right)
$$


for some polynomial $q_{i}$. To prove that this makes

$$
\left\{p_{0}\left(Q_{0}\right), Q_{i}-q_{1}\left(Q_{0}\right), \ldots, Q_{N-1}-q_{N-1}\left(Q_{0}\right)\right\}
$$

a polynomial explicit representation of the system (3-11)-(3-12) with respect to $Q_{0}$, there only remains to show that this set generates the same ideal as (3-11)-(3-12), or equivalently, that the latter ideal contains no univariate polynomial $\tilde{p}_{0}$ in $Q_{0}$ of degree smaller than $p_{0}$. If it were so, by considering the mapping $\alpha$ of Section $3 \mathrm{C}$, we would obtain the polynomial $\tilde{p}_{0}\left(h_{N}\right)$ in the ideal generated by $(2-4)-$ $(2-6)$, contradicting that $(3-15)$ is a polynomial explicit representation for this ideal.

One proves the converse similarly, using (3-13) instead of (3-14).

Since the Gröbner bases for both systems seem to be of the same "triangular" shape with a common univariate polynomial, the degree of this polynomial is a bound on the number of solutions. In all the cases $N=1, \ldots, 6$, it turns out to be of degree $2^{N-1}$. Besides, this number $2^{N-1}$ is also the product of the degrees of the polynomials in system (3-11)(3-12), the so-called Bézout bound of the system, which would bound the number of affine solutions if we could prove that this system has no solution at infinity [Cox et al. 1998].

Conjecture 3.10. Both systems of algebraic equations $(2-4)-(2-6)$ and $(3-11)-(3-12)$ have at most $2^{N-1}$ different solutions.

In order to elaborate on the Bézout bound of the system, we consider the system consisting of $(3-12)$ and the variant of $(3-11)$ obtained by setting its right-hand side to 0 . A proof that this system has no solution but the trivial solution

$$
Q_{0}=\cdots=Q_{N-1}=0
$$

would immediately imply by Bézout's theorem [Cox et al. 1998] that systems $(2-3)-(2-6)$ and (3-11)(3-12) both have exactly $2^{N-1}$ complex solutions counted with multiplicity. In other words, this would prove Conjecture 3.10 and the zero-dimensionality in Conjecture 3.1.

To corroborate Conjecture 3.10 on the number of solutions, we also performed the calculations of Gröbner bases for a total degree order up to $N=34$. With this choice of an order, the bases computed do not have the elimination property and the triangular shape discussed in Section 3, but suffice to derive the dimension and degree of the algebraic systems. To this end we used the specialized software Gb [1994], with which each basis was obtained in a matter of seconds. (We could not go further due to a limitation in the size of integers.) The result is that in each case the variety has dimension 0 and degree $2^{N-1}$, which proves the conjecture up to $N=34$.

Also here more seems to be true. For instance, up to $N=6$ the common univariate Gröbner basis polynomial always has $2^{N-1}$ different solutions. In particular, we have two real solutions in the cases $N=2$ and $N=3$; four real solutions in the cases $N=4$ and $N=5$; and eight real solutions if $N=6$. In the sidebar on page 82 we give the Mathematica procedure we have used together with some Gröbner bases output. Univariate polynomials in indeterminates other than $h_{N}$ or $Q_{0}$ have been obtained by further extensive calculations for $2 \leq N \leq 6$ (see Table 2). The observation is that for each $h_{i}$ and each $Q_{i}$ but $Q_{N-1}$, the degree of the univariate polynomial is $2^{N-1}$, whereas for $Q_{N-1}$ it is only 2 .

To end this section, we display two of the four solutions corresponding to the case $N=3$. In order to obtain those, first one has to find all solutions of the univariate Gröbner basis polynomial, which is

$$
p_{1}\left(x_{1}\right)=9-96 x_{1}-1536 x_{1}^{2}-4096 x_{1}^{3}+16384 x_{1}^{4}
$$

From this, one computes the two real solutions: a first real value for the list $\left(h_{-2}, h_{-1}, h_{0}, h_{1}, h_{2}, h_{3}\right)$ is

$$
\begin{aligned}
& \left(\frac{1+\sqrt{10}+\sqrt{5+2 \sqrt{10}}}{16}, \frac{5+\sqrt{10}+3 \sqrt{5+2 \sqrt{10}}}{16}, \frac{5-\sqrt{10}+\sqrt{5+2 \sqrt{10}}}{8},\right. \\
& \left.\frac{5-\sqrt{10}-\sqrt{5+2 \sqrt{10}}}{8}, \frac{5+\sqrt{10}-3 \sqrt{5+2 \sqrt{10}}}{16}, \frac{1+\sqrt{10}-\sqrt{5+2 \sqrt{10}}}{16}\right),
\end{aligned}
$$

and a second one is obtained by reflection. The four real solutions obtained for $N=4$ are also expressible as explicit expressions in nested radicals, but are too large to be displayed here.

The presence of $2^{N-1}$ different solutions that can be expressed in terms of nested square roots for $2 \leq$ $N \leq 4$ suggests that this could hold for all $N$. However, Klappenecker seemingly proved by a Galoistheoretic result that the scaling coefficients of the Daubechies wavelet cannot be expressed by radicals for all $N$ between 6 and 100 [Klappenecker 1997]. 


\section{WAVELETS ON THE INTERVAL}

\section{A. Meyer's Construction}

To our knowledge the first construction of orthogonal wavelets on the interval was proposed by Meyer [Meyer 1991]. His construction restricts compactly supported orthonormal wavelets on $\mathbb{R}$ (as considered in Section 2) to the interval $I:=[0,1]$ and manipulates the restricted functions in such a way that they form an orthonormal basis on $I$. Following his construction we develop a matrix analytical approach that allows to unify several constructions of wavelets on the interval in the same framework.

To avoid notational difficulties we restrict our attention to the construction of wavelets on $\mathbb{R}^{+}:=$ $[0, \infty)$. From our presentation it will become evident how the construction can be generalized to obtain a wavelet basis for $L^{2}(I)$.

We introduce the family of scaling functions restricted to $\mathbb{R}^{+}$

$$
\varphi_{m, k}^{\text {half }}(x):= \begin{cases}0 & \text { if } x<0 \\ \varphi_{m, k}(x) & \text { if } x \geq 0\end{cases}
$$

and the corresponding spaces

$$
V_{m}^{\text {half }}:=\overline{\operatorname{span}\left\{\varphi_{m, k}^{\text {half }}: k \in \mathbb{Z}\right\}}
$$

The spaces $V_{m}^{\text {half }}$ form a multiresolution analysis for $L^{2}\left(\mathbb{R}^{+}\right)$. The corresponding wavelet spaces $W_{m}^{\text {half }}$ are given by

$$
W_{m}^{\text {half }}:=\left(V_{m}^{\text {half }}\right)^{\perp} \cap V_{m-1}^{\text {half }} .
$$

As a consequence, we have the relation $V_{m-1}=$ $V_{m} \oplus W_{m}$, where the direct sum is orthogonal. By $P_{W_{m}^{\text {half }}}$ and $P_{V_{m}^{\text {half }}}$ we denote the orthogonal projection operators onto the spaces $W_{m}^{\text {half }}$ and $V_{m}^{\text {half }}$, respectively.

Since the scaling function $\varphi$ has support in the interval $[1-N, N]$, we have $\varphi_{m, k}^{\text {half }}=0$ for $k \leq-N$ and $\varphi_{m, k}^{\text {half }}=\varphi_{m, k}$ for $k \geq N-1$.

Wavelets on $\mathbb{R}^{+}$can be constructed in the following way:

1. Orthonormalize the set of functions $\left\{\varphi_{m, k}^{\text {half }}: k \geq\right.$ $1-N\}$. The obtained orthonormal basis of $V_{m}^{\text {half }}$ is denoted by $\left\{\varphi_{m, k}^{\text {edge }}: k \geq 1-N\right\}$.

2. Compute $P_{W_{m}^{\text {half }}} \varphi_{m-1, k}^{\text {edge }}$ and orthonormalize them to obtain an orthonormal basis $\psi_{m, k}^{\text {edge }}$ of $W_{m}^{\text {half }}$.
Orthonormalized scaling functions $\varphi_{\mathrm{m}, \mathrm{k}}^{\text {half }}$. The functions $\varphi_{m, k}^{\text {half }}$ are orthonormalized by making a basis transformation

$$
\varphi_{m}^{\mathrm{edge}}=A \varphi_{m}^{\text {half }}
$$

where

$$
\varphi_{m}^{\text {edge }}:=\left(\begin{array}{c}
\varphi_{m,-N+1}^{\text {edge }} \\
\varphi_{m,-N+2}^{\text {edge }} \\
\vdots
\end{array}\right), \quad \varphi_{m}^{\text {half }}:=\left(\begin{array}{c}
\varphi_{m,-N+1}^{\text {half }} \\
\varphi_{m,-N+2}^{\text {half }} \\
\vdots
\end{array}\right) .
$$

Using the notation

$$
\chi \chi^{t}:=\left(\left\langle\chi_{k}, \chi_{l}\right\rangle\right)_{k, l \geq 1-N}
$$

for any vector $\chi$, we see that the orthonormality of the functions $\varphi_{m}^{\text {edge }}$ is equivalent to the matrix equation

$$
\varphi_{m}^{\text {edge }} \varphi_{m}^{\text {edge }^{t}}=I
$$

From (4-2) it follows that

$$
I=A \varphi_{m}^{\text {half }} \varphi_{m}^{\text {half }}{ }^{t} A^{t}
$$

If the matrix

$$
\Lambda:=\varphi_{m}^{\text {half }} \varphi_{m}^{\text {half }}{ }^{t}
$$

of the inner products of the truncated scaling functions $\varphi_{m, k}^{\text {half }}$ is known, then the matrix $A$ in $(4-2)$ can be obtained by the Cholesky factorization

$$
\Lambda=\left(A^{-1}\right)\left(A^{-1}\right)^{t}
$$

where $A^{-1}$ is regular and of lower triangular form. Therefore the matrix $A$ is also lower triangular. In view of (4-2) and of the supports of the functions $\varphi_{m, l}^{\text {half }}$, this in particular ensures staggered support of the functions $\varphi_{m, k}^{\text {edge }}$; that is,

$$
\operatorname{supp} \varphi_{m, k}^{\text {edge }} \subseteq\left[0,2^{m}(N+k)\right] .
$$

We now derive the refinement equations (similar to $(2-1)$ and $(2-2))$ for $\varphi_{m}^{\text {edge }}$ and the corresponding wavelets $\psi_{m}^{\text {edge }}$. These equations are the basis for the implementation of multiresolution cascade algorithms [Mallat 1989], as they are used in data compression; see [Williams and Amaratunga 1994], for example.

By truncation of the dilation equations (2-1), the truncated scaling functions $\varphi_{m, k}^{\text {half }}$ satisfy the dilation equation

$$
\varphi_{m, k}^{\text {half }}=\frac{1}{\sqrt{2}} \sum_{r \in \mathbb{Z}} h_{r-2 k} \varphi_{m-1, r}^{\text {half }}
$$


which in matrix form rewrites

$$
\varphi_{m}^{\text {half }}=H \varphi_{m-1}^{\text {half }} .
$$

Here the dilation matrix $H$ makes the transition from the truncated scaling functions at scale $m-1$ to those at the finer scale $m$, and is therefore called the refinement matrix. This is a $1 \times 2$ block-Toeplitz matrix:

$$
H_{k, l}=\left\{\begin{array}{cl}
\frac{h_{l-2 k}}{\sqrt{2}} & \text { if } 1-N \leq l-2 k \leq N, \\
0 & \text { otherwise }
\end{array}\right.
$$

in other words, $H_{k, l}$ is 0 outside a band of slope $\frac{1}{2}$. From (4-2) and (4-5) it follows that

$$
\varphi_{m}^{\text {edge }}=A \varphi_{m}^{\text {half }}=A H \varphi_{m-1}^{\text {half }}=A H A^{-1} \varphi_{m-1}^{\text {edge }} .
$$

Thus the refinement matrix $H^{\text {edge }}$ for the dilation equation of the edge scaling functions $\varphi_{m}^{\text {edge }}$ is

$$
H^{\text {edge }}=A H A^{-1} \text {. }
$$

Note that $H^{\text {edge }}$ is no longer a $1 \times 2$ block-Toeplitz matrix (which is the case for wavelets on $\mathbb{R}$ ). This reflects the fact that the edge scaling functions cannot be obtained as shifts of a single function.

Projection onto the Subspace $W_{m}^{\text {half }}$. Now we construct the edge wavelets and derive their refinement matrix. The projections of $\varphi_{m-1, k}^{\text {edge }}$ onto $W_{m}^{\text {half }}$, which was defined by (4-1), are given by

$$
\begin{aligned}
\psi_{m, k}^{\text {half }} & :=P_{W_{m}^{\text {half }}} \varphi_{m-1, k}^{\text {edge }} \\
& =\varphi_{m-1, k}^{\text {edge }}-\sum_{l}\left\langle\varphi_{m-1, k}^{\text {edge }}, \varphi_{m, l}^{\text {edge }}\right\rangle \varphi_{m, l}^{\text {edge }},
\end{aligned}
$$

or equivalently with matrices

$$
\psi_{m}^{\text {half }}=\varphi_{m-1}^{\text {edge }}-\left(\varphi_{m-1}^{\text {edge }} \varphi_{m}^{\text {edge }}{ }^{t}\right) \varphi_{m}^{\text {edge }} .
$$

In view of (4-6) and (4-7) it follows that

$$
\begin{aligned}
\psi_{m}^{\text {half }} & =\varphi_{m-1}^{\text {edge }}-\left(\varphi_{m-1}^{\text {edge }}\left(\varphi_{m-1}^{\text {edge }}{ }^{t} H^{\text {edge }^{t}}\right)\right) H^{\text {edge }} \varphi_{m-1}^{\text {edge }} \\
& =\left(I-H^{\text {edge }^{t}} H^{\text {edge }}\right) \varphi_{m-1}^{\text {edge }}=: G^{\text {half }} \varphi_{m-1}^{\text {edge }} .
\end{aligned}
$$

The matrix $G^{\text {half }}$ does not have $1 \times 2$ lower blocktriangular form, i.e., it does not fulfill $G_{k, l}^{\text {half }}=0$ for $l>N+2 k$. Consequently the functions $\psi_{m, k}^{\text {half }}$ do not have staggered support. In [Cohen et al. 1993b] it is established that there exists a basis transformation $U$ such that

$$
\psi_{m}^{\text {stag }}:=U \psi_{m}^{\text {half }}
$$

has staggered support. In Section $4 \mathrm{E}$ we give a simple constructive algorithm for calculating $U$. The functions $\psi_{m, k}^{\mathrm{stag}}$ are orthonormalized to get the edge wavelets

$$
\psi_{m}^{\text {edge }}=B \psi_{m}^{\text {stag }} .
$$

Orthonormalized edge wavelets $\psi_{\mathrm{m}, \mathrm{k}}^{\text {edge }}$. In the following we outline the orthonormalization procedure, i.e., the calculation of the matrix $B$. Let $\Lambda_{w}$ be the matrix of inner products of the functions $\psi_{m, k}^{\text {stag }}$. Then from (4A) and (4-9) it follows that

$$
\Lambda_{w}:=\psi_{m}^{\text {stag }} \psi_{m}^{\text {stagt }}=U G^{\text {half }} G^{\text {half }} U^{t} .
$$

On the other hand, we get from (4-10) and the orthonormality of the functions $\psi_{m}^{\text {edge }}$

$$
\Lambda_{w}=\left(B^{-1}\right)\left(B^{-1}\right)^{t} .
$$

Thus the matrix $B$ can be calculated from $\Lambda_{w}$ by a Cholesky factorization and inversion. From (4A)(4-10) it follows that

$$
\psi_{m}^{\text {edge }}=B U G^{\text {half }} \varphi_{m-1}^{\text {edge }} .
$$

Thus the matrix $G^{\text {edge }}$ for the refinement equation of the edge wavelets is given by

$$
G^{\text {edge }}=B U G^{\text {half }} .
$$

To make the calculations complete we have to determine the matrix $\Lambda$ in $(4-3)$. The matrix $\Lambda$ is independent of the scale $m$ as one can see from the following argument. Since

$$
\varphi_{m, k}^{\text {half }}(2 x)=\sqrt{2} \varphi_{m-1, k}^{\text {half }}(x)
$$

it follows that

$$
\begin{aligned}
\left\langle\varphi_{m-1, k}^{\text {half }}, \varphi_{m-1, l}^{\text {half }}\right\rangle & =2\left\langle\varphi_{m, k}^{\text {half }}(2 \cdot), \varphi_{m, l}^{\text {half }}(2 \cdot)\right\rangle \\
& =\left\langle\varphi_{m, k}^{\text {half }}, \varphi_{m, l}^{\text {half }}\right\rangle .
\end{aligned}
$$

In view of $(4-5)$ it follows that

$$
\Lambda=\varphi_{m}^{\text {half }} \varphi_{m}^{\text {half }^{t}}=H \Lambda H^{t} .
$$

Since $\Lambda_{k, l}=\left\langle\varphi_{m, k}^{\text {half }}, \varphi_{m, l}^{\text {half }}\right\rangle=\delta_{k, l}$ if $k \geq N-1$ or $l \geq$ $N-1$, equation (4-14) above can be reduced to

$$
\Lambda_{0}=H_{0} \Lambda_{0}^{\mathrm{ext}} H_{0}^{t},
$$

where $H_{0} \in \mathbb{R}^{(2 N-2) \times(4 N-4)}$ with $\left(H_{0}\right)_{k, l}=h_{l-2 k} / \sqrt{2}$ for $1-N \leq k \leq N-2$ and $1-N \leq l \leq 3 N-4$, and $\Lambda_{0}^{\text {ext }}$ extends the matrix $\Lambda_{0} \in \mathbb{R}^{(2 \bar{N}-2) \times(2 N-2)}$ in the form

$$
\Lambda_{0}^{\mathrm{ext}}=\left(\begin{array}{cc}
\Lambda_{0} & 0 \\
0 & I
\end{array}\right) \in \mathbb{R}^{(4 N-4) \times(4 N-4)}
$$


Equation (4-15) is a non-homogeneous linear system in as many unknowns as equations. Whereas it gets numerically ill-conditioned, it can be solved symbolically. We have strong evidence that there exists a solution $\Lambda_{0}$ of (4-15) but so far we have no proof: the existence of a solution is closely related to the eigenvalues of the matrix

$$
H_{1} \in \mathbb{R}^{(2 N-2) \times(2 N-2)}
$$

given by restriction of $H_{0}$ to the first $2 N-2$ columns. If the absolute values of the eigenvalues of $H_{1}$ are less than 1, then from (4-15) it follows that

$$
\Lambda_{0}=\sum_{n=0}^{\infty} H_{1}^{n} H_{2} H_{2}^{t}\left(H_{1}^{t}\right)^{n}
$$

where $\mathrm{H}_{2}$ are the last $2 \mathrm{~N}-2$ columns of $H_{0}$.

We mention a result from [Strang 1996], which indicates that $N$ eigenvalues of $H_{1}$ are given by

$$
2^{-k-1 / 2}, \text { for } k=0, \ldots, N-1 \text {. }
$$

Since there is no estimate for the other $N-1$ eigenvalues available, this result does not give existence of a solution. However, in all our considered examples the largest eigenvalue turned out to be $1 / \sqrt{2}$.

\section{B. The Construction of Cohen, Daubechies and Vial}

The starting point is again a compactly supported orthogonal wavelet family on $\mathbb{R}$. As in Meyer's approach, the construction of Cohen, Daubechies and Vial [Cohen et al. 1993b] retains the interior scaling functions and adds adapted edge scaling functions. That paper and [Cohen et al. 1993a] introduce the family of transformed scaling functions restricted to $\mathbb{R}^{+}$as follows:

$$
\varphi_{m, k}^{\text {mod }}=\left\{\begin{array}{cl}
\sum_{l}\left(\begin{array}{c}
N-1-l \\
N-1-k
\end{array}\right) \varphi_{m, l}^{\text {half }} & \text { if } 0 \leq k \leq N-1, \\
\varphi_{m, k}^{\text {half }} & \text { if } k \geq N
\end{array}\right.
$$

The functions $\varphi_{m, k}^{\bmod }$ generate all polynomials up to degree $N-1$ [Cohen et al. 1993b, Proposition 4.1]. In contrast to Meyer's construction this approach requires less edge scaling functions to fulfill this task. While in Meyer's construction the spaces $V_{m}^{\text {half }}$ are just the projections of $V_{m}$ onto $L^{2}\left(\mathbb{R}^{+}\right)$, here the space

$$
V_{m}^{\text {half }}:=\overline{\operatorname{span}\left\{\varphi_{m, k}^{\bmod }: k \in \mathbb{N}_{0}\right\}}=T\left(V_{m}\right),
$$

where $T=\left(T_{k, l}\right)$ is a matrix with indices $0 \leq k$ and $1-N \leq l$ that satisfies

$$
T_{k, l}=\left\{\begin{array}{cl}
\left(\begin{array}{c}
N-1-l \\
N-1-k
\end{array}\right) & \text { if } 0 \leq k \leq N-1, \\
\delta_{k, l} & \text { if } k \geq N
\end{array}\right.
$$

Since $T_{k, l}=0$ if $l>k$ the family

$$
\varphi_{m}^{\text {mod }}=T \varphi_{m}^{\text {half }}
$$

has staggered support. The spaces $V_{m}^{\text {half }}$ define a multiresolution analysis on $L^{2}\left(\mathbb{R}^{+}\right)$and the corresponding wavelet spaces are given by

$$
W_{m}^{\text {half }}:=\left(V_{m}^{\text {half }}\right)^{\perp} \cap V_{m-1}^{\text {half }} .
$$

The functions $\varphi_{m, k}^{\text {mod }}$ can be orthonormalized by a basis transformation

$$
\varphi_{m}^{\mathrm{edge}}=A \varphi_{m}^{\mathrm{mod}} .
$$

Again the orthonormalization matrix $A$ is determined by the Cholesky decomposition of

$$
\tilde{\Lambda}:=\varphi_{m}^{\bmod } \varphi_{m}^{\bmod t}=T \Lambda T^{t},
$$

where $\Lambda$ is as in $(4-3)$, i.e.,

$$
\tilde{\Lambda}=\left(A^{-1}\right)\left(A^{-1}\right)^{t} .
$$

We now determine the filter matrix $H^{\text {edge}}$; once the filter matrix $H^{\text {edge }}$ is constructed, the refinement matrix $G^{\text {edge }}$ of the edge wavelets can be calculated analogously to the construction presented in Section $4 \mathrm{~A}$.

The filter matrix for the dilation equation satisfies

$$
\varphi_{m}^{\text {edge }}=H^{\text {edge }} \varphi_{m-1}^{\text {edge }} .
$$

From (4-16) and (4-5) we get

$$
\varphi_{m}^{\text {mod }}=T \varphi_{m}^{\text {half }}=T H \varphi_{m-1}^{\text {half }} .
$$

Suppose that there exists a dilation equation for $\varphi_{m}^{\text {mod }}$, i.e.,

$$
\varphi_{m}^{\bmod }=H^{\bmod } \varphi_{m-1}^{\bmod },
$$

then from (4-21) and (4-22) it follows that

$$
T H=H^{\mathrm{mod}} T .
$$

Multiplication of this equation by a right inverse $T^{\dagger}$ of $T$ from the right gives

$$
H^{\mathrm{mod}}=T H T^{\dagger} .
$$


This yields the following condition on $T, H$ and $T^{\dagger}$ :

$$
T H T^{\dagger} T=T H,
$$

which is equivalent to

$$
\mathcal{N}(T) \subset \mathcal{N}(T H)
$$

where $\mathcal{N}$ denotes the nullspace. In particular this shows that the condition (4-24) is independent on the choice of the right inverse $T^{\dagger}$.

From (4-23) and (4-20) it follows that

$$
H^{\text {edge }}=A T H T^{\dagger} A^{-1} .
$$

The rest of procedure to construct $G^{\text {edge }}$ is analogous as in Section 4A. For the reader's convenience we have summarized the calculation of the refinement matrices in Section 4E.

This matrix analytical approach clearly reveals the similarity between the constructions proposed by Meyer and Cohen, Daubechies and Vial. In fact the only difference in both constructions is that the construction of the filter matrix $H^{\text {edge }}$ incorporates the matrix $T$. Any right-invertible matrix $T$ satisfying (4-25) can be used to construct wavelets on $\mathbb{R}^{+}$ with different properties. The special form of the matrix $T$ proposed in [Cohen et al. 1993b] guarantees that the scaling functions have staggered support and that any polynomial up to degree $N-1$ can be represented as a linear combination of the scaling functions. Setting $T=I$ gives the construction proposed by Meyer.

\section{C. The Biorthogonal Case: The Constructions of Dahmen et al.}

In this section we show that the our matrix approach for the construction of wavelets on the interval can be generalized in a natural way to the construction of biorthogonal wavelets on the interval. This outlines the constructions proposed in [Dahmen et al. 1999; Dahmen and Schneider 1998].

In the biorthogonal case one requires two scaling functions $\varphi$ and $\tilde{\varphi}$ satisfying dilation equations

$$
\begin{aligned}
& \varphi(x)=\sum_{k=1-N}^{N} h_{k} \varphi(2 x-k), \\
& \tilde{\varphi}(x)=\sum_{k=1-\tilde{N}}^{\tilde{N}} \tilde{h}_{k} \tilde{\varphi}(2 x-k) .
\end{aligned}
$$

Both scaling functions satisfy $(2-4),(2-6)$ and are biorthogonal, i.e.,

$$
\sum_{k} h_{k} \tilde{h}_{k-2 l}=2 \delta_{0, l}
$$

The corresponding multiresolution analyses are given by

$$
\begin{aligned}
& V_{m}:=\overline{\operatorname{span}\left\{\varphi_{m, k}: k \in \mathbb{Z}\right\}}, \\
& \tilde{V}_{m}:=\overline{\operatorname{span}\left\{\tilde{\varphi}_{m, k}: k \in \mathbb{Z}\right\}} .
\end{aligned}
$$

The wavelet spaces $W_{m}$ and $\tilde{W}_{m}$ are then defined by

$$
W_{m}=V_{m-1} \cap \tilde{V}_{m}^{\perp}, \quad \tilde{W}_{m}=\tilde{V}_{m-1} \cap V_{m}^{\perp} .
$$

For more background on biorthogonal wavelets see [Cohen et al. 1992].

Following the notation of the previous sections we define the modified scaling functions on $\mathbb{R}^{+}$by

$$
\varphi_{m}^{\text {mod }}=T \varphi_{m}^{\text {half }} \quad \text { and } \quad \tilde{\varphi}_{m}^{\text {mod }}=\tilde{T} \tilde{\varphi}_{m}^{\text {half }},
$$

where again $\varphi_{m}^{\text {half }}$ and $\tilde{\varphi}_{m}^{\text {half }}$ are the restrictions to the positive real line.

The two families $\varphi_{m}^{\text {mod }}$ and $\tilde{\varphi}_{m}^{\text {mod }}$ are biorthogonalized by two basis transforms $A$ and $\tilde{A}$, i.e.,

$$
\varphi_{m}^{\text {edge }}:=A \varphi_{m}^{\text {mod }} \quad \text { and } \quad \tilde{\varphi}_{m}^{\text {edge }}:=\tilde{A} \tilde{\varphi}_{m}^{\text {mod }}
$$

satisfy

$$
\varphi_{m}^{\text {edge }} \tilde{\varphi}_{m}^{\text {edge }}{ }^{t}=I
$$

Analogously to $(4-18),(4-19)$ the last equation is equivalent to

$$
\left(A^{-1}\right)\left(\tilde{A}^{-1}\right)^{t}=T \Lambda \tilde{T}^{t}
$$

where $\Lambda:=\varphi_{m}^{\text {half }} \tilde{\varphi}_{m}^{\text {half }}{ }^{t}$.

For a given matrix $T \Lambda \tilde{T}^{t}$ the factorization into the matrices $A^{-1}$ and $\tilde{A}^{-1}$ can be computed in several ways: one could for example use a factorization by means of a SVD as suggested in [Dahmen et al. 1999], an $L U$-decomposition, or simply set $A=I$ and $\tilde{A}=\left(T \Lambda \tilde{T}^{t}\right)^{-1}$. Each possible factorization results in different biorthogonal bases for the same multiresolution spaces $V_{m}^{\text {half }}$ and $\tilde{V}_{m}^{\text {half }}$. For orthogonal wavelets we calculated the factorization by a Cholesky decomposition.

The matrix $\Lambda$ can be calculated similarly to the orthogonal case (see (4-14)) as the solution of the following linear inhomogeneous system:

$$
\Lambda=H \Lambda \tilde{H}^{t} .
$$


The dilation matrices $H^{\text {edge }}$ and $\tilde{H}^{\text {edge }}$ are given by $H^{\text {edge }}=A T H T^{\dagger} A^{-1} \quad$ and $\quad \tilde{H}^{\text {edge }}=\tilde{A} \tilde{T} \tilde{H} \tilde{T}^{\dagger} \tilde{A}^{-1}$, where $T^{\dagger}$ and $\tilde{T}^{\dagger}$ denote the right inverses of $T$ and $\tilde{T}$ satisfying

$$
\mathcal{N}(T) \subset \mathcal{N}(T H) \quad \text { and } \quad \mathcal{N}(\tilde{T}) \subset \mathcal{N}(\tilde{T} \tilde{H})
$$

Note the similarity of the constructions of $H^{\text {edge }}$ in the orthogonal and biorthogonal case!

The construction of the biorthogonal wavelet bases can be carried over from the orthogonal case. Since

$$
V_{m} \oplus W_{m}=V_{m-1} \quad \text { and } \quad \tilde{V}_{m} \oplus \tilde{W}_{m}=\tilde{V}_{m-1}
$$

we can write the projections of $\varphi_{m-1}^{\text {edge }}$ and $\tilde{\varphi}_{m-1}^{\text {edge }}$ onto $W_{m}$ and $\tilde{W}_{m}$ as

$$
\begin{aligned}
& \psi_{m}^{\text {half }}:=P_{W_{m}} \varphi_{m-1}^{\text {edge }}=\varphi_{m-1}^{\text {edge }}-P_{V_{m}} \varphi_{m-1}^{\text {edge }}, \\
& \tilde{\psi}_{m}^{\text {half }}:=P_{\tilde{W}_{m}} \tilde{\varphi}_{m-1}^{\text {edge }}=\tilde{\varphi}_{m-1}^{\text {edge }}-P_{\tilde{V}_{m}} \tilde{\varphi}_{m-1}^{\text {edge }},
\end{aligned}
$$

and consequently

$$
\psi_{m}^{\text {half }}=G^{\text {half }} \varphi_{m-1}^{\text {edge }} \quad \text { and } \quad \tilde{\psi}_{m}^{\text {half }}=\tilde{G}^{\text {half }} \tilde{\varphi}_{m-1}^{\text {edge }},
$$

where

$$
\begin{aligned}
& G^{\text {half }}=I-\tilde{H}^{\text {edge }{ }^{t}} H^{\text {edge }}, \\
& \tilde{G}^{\text {half }}=I-H^{\text {edge }{ }^{t}} \tilde{H}^{\text {edge }} .
\end{aligned}
$$

In order to biorthogonalize the families of functions $\psi_{m}^{\text {half }}$ and $\tilde{\psi}_{m}^{\text {half }}$ we set

$$
\psi_{m}^{\text {edge }}:=B \psi_{m}^{\text {half }} \quad \text { and } \quad \tilde{\psi}_{m}^{\text {edge }}:=\tilde{B} \tilde{\psi}_{m}^{\text {half }},
$$

where the matrices $B$ and $\tilde{B}$ satisfy

$$
\left(B^{-1}\right)\left(\tilde{B}^{-1}\right)^{t}=\Lambda_{w}
$$

where

$$
\Lambda_{w}:=\psi_{m}^{\text {half }} \tilde{\psi}_{m}^{\text {half }}{ }^{t}=G^{\text {half }} \tilde{G}^{\text {half }}{ }^{t} .
$$

The construction just presented reveals that there is more freedom in generating biorthogonal wavelets on $\mathbb{R}^{+}$than for the the construction of orthogonal wavelets. The choice of the matrices $T$ and $\tilde{T}$ determines the properties of the multiresolution analyses. As in the orthogonal case, any $T$ and $\tilde{T}$, compatible with $H$ and $\tilde{H}$ in the sense of $(4-30)$ can be used to construct biorthogonal wavelets on the interval. The choices of the biorthogonalizations (4-28) and (4-31) affect the scaling functions and wavelets, but not the multiresolution and wavelet spaces. Dahmen et al. [1999] suggested transformations $T$ and $\tilde{T}$ for the construction of biorthogonal wavelet bases with certain polynomial exactness.

\section{D. (Bi-)orthogonal Wavelets with Staggered Support}

The matrix analytical point of view of constructing wavelets on the half line clearly indicates how to impose additional properties on the wavelets and scaling functions. In the construction above we have not paid any attention to preserve staggered support of the scaling functions and wavelets. In the following we show how to construct (bi-)orthogonal wavelets and scaling functions with staggered support. To our knowledge biorthogonal wavelets on the interval with staggered support have not been considered in the literature so far.

The following lemma guarantees existence of a ba$\operatorname{sis} \varphi_{m}^{\text {stag }}$ of $V_{m}^{\text {half }}$ with staggered support.

Lemma 4.1. Let $T_{0} \in \mathbb{R}^{K \times(2 N-1)}, K \leq 2 N-1$ and let $T_{0,1}$ be the $K \times K$ submatrix consisting of the last $K$ columns of $T_{0}$. If $T_{0,1}$ is invertible, then there exists an invertible matrix $S_{0} \in \mathbb{R}^{K \times K}$ such that $S_{0} T_{0}$ is of lower triangular form.

Proof. Since $T_{0,1}$ is invertible, $\left(T_{0,1}^{-1}\right)^{t}$ exists and can be decomposed by an $L U$-factorization into

$$
P\left(T_{0,1}^{-1}\right)^{t}=L U,
$$

where $L$ and $U$ are lower and upper triangular matrices, respectively, and $P$ is a permutation matrix. Thus

$$
L^{t} P^{t} T_{0,1}=\left(U^{-1}\right)^{t}
$$

is a lower triangular matrix. (Note that $U^{t}$ is lower triangular, thus also $\left(U^{-1}\right)^{t}$, and $L^{t} P^{t}$ is invertible.) Let $T_{0}=\left(T_{0,0}, T_{0,1}\right)$, then as a consequence

$$
L^{t} P^{t} T_{0}=L^{t} P^{t}\left(T_{0,0}, T_{0,1}\right)=\left(L^{t} P^{t} T_{0,0},\left(U^{-1}\right)^{t}\right)
$$

is lower triangular. Thus the assertion is proved with $S_{0}:=L^{t} P^{t}$.

$T$ in $(4-16)$ is of the form

$$
T=\left(\begin{array}{cc}
T_{0} & 0 \\
0 & I
\end{array}\right) .
$$

Let $S_{0}$ be defined as in the lemma above. Then

$$
T^{\text {stag }}:=\left(\begin{array}{cc}
S_{0} & 0 \\
0 & I
\end{array}\right)\left(\begin{array}{cc}
T_{0} & 0 \\
0 & I
\end{array}\right)=\left(\begin{array}{cc}
S_{0} T_{0} & 0 \\
0 & I
\end{array}\right)
$$

is of lower triangular form and so $\varphi_{m}^{\text {stag }}:=T^{\text {stag }} \varphi_{m}^{\text {half }}$ has staggered support. Proposition 4.3 in [Cohen et al. 1993b] guarantees the existence of wavelets with staggered support. 
The above considerations can be easily carried over to biorthogonal wavelets. In order to get biorthogonal wavelets and scaling functions with staggered support an $L U$-factorization of (4-29) has to be performed, since only this factorization guarantees that the staggered support is preserved during the biorthogonalization procedure.

\section{E. An Algorithm for the Calculation of the Refinement Matrices}

For the reader's convenience we summarize the computational steps for calculating the refinement matrices $H^{\text {edge }}$ and $G^{\text {edge }}$ in the orthogonal case. The modifications of this algorithm to calculate the refinement matrices in the biorthogonal case are obvious.

Each step of the proposed algorithm can either be performed numerically or symbolically.

The data are the filter sequence $h_{k}$ of a compactly supported orthonormal wavelet family on $\mathbb{R}$ with $h_{k}=0$ if $k \leq-N$ or $k \geq N+1$ and the matrix $T \in \mathbb{R}^{K \times(2 N-1)}$. (We have $T=I \in \mathbb{R}^{(2 N-1) \times(2 N-1)}$ for Meyer's construction and

$$
\left(T_{k, l}\right)=\left(\begin{array}{c}
N-l-1 \\
N-k-1
\end{array}\right) \in \mathbb{R}^{N \times(2 N-1)}
$$

for the construction of Cohen et al.)

1. Define the filter matrix

$$
H:=\left(H_{k, l}\right) \in \mathbb{R}^{(2 N-1) \times(4 N-2)},
$$

with

$$
H_{k, l}=h_{l-2 k} / \sqrt{2}
$$

for $1-N \leq k \leq N-1$ and $1-N \leq l \leq 3 N-2$.

2. Solve

$$
\Lambda=H \Lambda^{\mathrm{ext}} H^{t}
$$

with $\Lambda \in \mathbb{R}^{(2 N-1) \times(2 N-1)}$ and

$$
\Lambda^{\mathrm{ext}}=\left(\begin{array}{cc}
\Lambda & 0 \\
0 & I
\end{array}\right) \in \mathbb{R}^{(4 N-2) \times(4 N-2)} .
$$

3. Compute the matrix of inner products

$$
\tilde{\Lambda}=T \Lambda T^{t} \in \mathbb{R}^{K \times K} .
$$

4. Compute $A \in \mathbb{R}^{K \times K}$ from the Cholesky decomposition

$$
\tilde{\Lambda}=\left(A^{-1}\right)\left(A^{-1}\right)^{t} .
$$

5. The dilation matrix for the edge scaling functions is then given by

$$
H^{\text {edge }}=A T H\left(T^{\text {ext }}\right)^{\dagger}\left(A^{\text {ext }}\right)^{-1} \in \mathbb{R}^{K \times(K+2 N-1)},
$$

where

$$
A^{\mathrm{ext}}=\left(\begin{array}{cc}
A & 0 \\
0 & I
\end{array}\right) \in \mathbb{R}^{(K+2 N-1) \times(K+2 N-1)} .
$$

and

$$
T^{\mathrm{ext}}=\left(\begin{array}{ll}
T & 0 \\
0 & I
\end{array}\right) \in \mathbb{R}^{(K+2 N-1) \times 4 N-2},
$$

and $\left(T^{\mathrm{ext}}\right)^{\dagger}$ is a right inverse of $T^{\mathrm{ext}}$.

6. Compute

$$
C=\left(I-H^{\text {edge }^{t}} H^{\text {edge }}\right) \in \mathbb{R}^{(K+2 N-1) \times(K+2 N-1)},
$$

and define $G^{\text {half }} \in \mathbb{R}^{N \times(K+2 N-1)}$ as

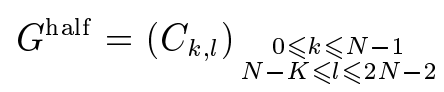

7. Compute an upper triangular matrix

$$
U \in \mathbb{R}^{(N-1) \times(N-1)}
$$

such that $U G^{\text {half }}$ is a lower triangular block matrix in the sense that $\left(U G^{\text {half }}\right)_{k, l}=0$ for $l>$ $N+2 k$. This can be done using the following algorithm:

a. Define the matrix $\tilde{C} \in \mathbb{R}^{N \times N}$ by

$$
\tilde{C}_{k, l}=G_{k, N+2 l}^{\text {half }}, \text { for } 0 \leq k, l \leq N-1 .
$$

b. Compute $U$ by the unpivoted $L U$-decomposition

$$
\tilde{C}^{-1}=L U \text {. }
$$

8. Compute the matrix of inner products

$$
\Lambda_{w}=U G^{\text {half }} G^{\text {half }^{t}} U^{t} \in \mathbb{R}^{N \times N} .
$$

9. Compute the Cholesky decomposition

$$
\Lambda_{w}=\left(B^{-1}\right)\left(B^{-1}\right)^{t} .
$$

10. The filter matrix $G^{\text {edge }}$ is then given by

$$
G^{\text {edge }}=B U G^{\text {half }} .
$$

The entries of $H^{\text {edge }}$ and $G^{\text {edge }}$ for $k \geq N$ are given by

$$
H_{k, l}^{\text {edge }}=h_{l-2 k} / \sqrt{2} \quad \text { and } \quad G_{k, l}^{\text {edge }}=g_{l-2 k} / \sqrt{2} .
$$

\section{EXPLICIT RESULTS}

This section presents some explicit results for cases of particular interest. We start with the closed form representations of the filter coefficients for the Daubechies wavelets, shown on the next page. 


\section{Function definitions}

Polys1 $\left[N_{-}\right]:=\left\{\operatorname{Sum}\left[Q[N, j] / 2^{\wedge} j,\{j, 0, N-1\}\right]-1 / 2^{\wedge}(2 N-2)\right\}$

Polys2 [N_] :=Table [Sum [Binomial $[4 N-i-j-2,2 N+21-i-1] * Q[N, i] * Q[N, j],\{i, 0, N-1\},\{j, 0, N-1\}]$, $\{1,1, N-1\}]$

AllPolys [N_] :=Join[Polys $1[\mathrm{~N}]$, Polys2 [N] $]$

Eqns $\left[N_{-}\right]:=$Map [\#==0\&,AllPolys $\left.[\mathrm{N}]\right]$

Unknowns $\left[N_{-}\right]:=$Table $[Q[N, j],\{j, 0, N-1\}]$

CFSols [N_] :=Solve [Eqns [N], Unknowns [N]]

GB [N_] : =GroebnerBasis [AllPolys [N] , Reverse [Unknowns [N]]]

$\operatorname{cc}\left[\mathrm{N}_{-}, \mathrm{k}_{-}\right]:=$Binomial $[2 \mathrm{~N}-1, \mathrm{~N}-\mathrm{k}] * \operatorname{Sum}[\mathrm{Q}[\mathrm{N}, \mathrm{j}]$ Binomial $[\mathrm{N}-\mathrm{k}, \mathrm{j}] / \operatorname{Binomial}[2 \mathrm{~N}-1, j],\{\mathrm{j}, 0, \mathrm{~N}-1\}]$

CoefficientTable[N ,rules_ $]:=$ Table[h[N,k]->Simplify $[\mathrm{cc}[\mathrm{N}, \mathrm{k}] /$.rules $],\{\mathrm{k}, 1-\mathrm{N}, \mathrm{N}\}]$

\section{Gröbner basis in the case $\mathrm{N}=3$}

GB [3]

$$
\begin{aligned}
& \left\{9-96 Q(3,0)-1536 Q(3,0)^{2}-4096 Q(3,0)^{3}+16384 Q(3,0)^{4},\right. \\
& 21 Q(3,0)+32 Q(3,0)^{2}-128 Q(3,0)^{3}+3 Q(3,1), \\
& \left.-3-120 Q(3,0)-256 Q(3,0)^{2}+1024 Q(3,0)^{3}+12 Q(3,2)\right\}
\end{aligned}
$$

\section{A real solution for $\mathrm{N}=3$}

rules $=$ Simplify $[$ CFSols $[3][[3]]]$

CoefficientTable [3, rules]

$$
\begin{array}{rlrl}
\{h(3,-2) & \rightarrow \frac{1+\sqrt{10}+\sqrt{5+2 \sqrt{10}}}{16}, & h(3,-1) & \rightarrow \frac{5+\sqrt{10}+3 \sqrt{5+2 \sqrt{10}}}{16}, \\
h(3,0) & \rightarrow \frac{5-\sqrt{10}+\sqrt{5+2 \sqrt{10}}}{8}, & h(3,1) & \rightarrow \frac{5-\sqrt{10}-\sqrt{5+2 \sqrt{10}}}{8}, \\
h(3,2) & \rightarrow \frac{5+\sqrt{10}-3 \sqrt{5+2 \sqrt{10}}}{16}, & \left.h(3,3) \rightarrow \frac{1+\sqrt{10}-\sqrt{5+2 \sqrt{10}}}{16}\right\}
\end{array}
$$

\section{Gröbner basis in the case $\mathrm{N}=4$}

GB $[4]$

$$
\begin{aligned}
& \left\{625+16000 Q(4,0)-1433600 Q(4,0)^{2}+22937600 Q(4,0)^{3}+220200960 Q(4,0)^{4}\right. \\
& \quad-4697620480 Q(4,0)^{5}-60129542144 Q(4,0)^{6}-137438953472 Q(4,0)^{7}+1099511627776 Q(4,0)^{8}, \\
& 125+389200 Q(4,0)-1469440 Q(4,0)^{2}-29245440 Q(4,0)^{3}+124780544 Q(4,0)^{4} \\
& \quad+2936012800 Q(4,0)^{5}+7516192768 Q(4,0)^{6}-51539607552 Q(4,0)^{7}+39200 Q(4,1), \\
& -1875-6661200 Q(4,0)+57164800 Q(4,0)^{2}+775864320 Q(4,0)^{3}-9064939520 Q(4,0)^{4} \\
& \quad-136113553408 Q(4,0)^{5}-323196289024 Q(4,0)^{6}+2456721293312 Q(4,0)^{7}+196000 Q(4,2), \\
& -11625+3553200 Q(4,0)-42470400 Q(4,0)^{2}-483409920 Q(4,0)^{3}+7817134080 Q(4,0)^{4} \\
& \left.\quad+106753425408 Q(4,0)^{5}+248034361344 Q(4,0)^{6}-1941325217792 Q(4,0)^{7}+98000 Q(4,3)\right\}
\end{aligned}
$$

Mathematica program to calculate the filter coefficients of the Daubechies wavelets. The lines in this font (flush left) represent input typed to the computer. 
Next we provide explicit values for the univariate polynomials that describe systems $(2-4)-(2-6)$ and (3-11)-(3-12), as described in Sections $3 \mathrm{~A}$ and 3 D. Specifically, we choose to give the univariate polynomial satisfied by $h_{N}$, or $x_{1}$ in the notation of Section 3A, for any solution of $(2-4)-(2-6)$, which is the same as the univariate polynomial satisfied by $Q_{0}$ for any solution of (3-11)-(3-12). For fixed $N$, univariate polynomials in another $h_{i}$ or $Q_{i}$ (except for $Q_{0}$ ) would be of the same degree and with integer coefficients of same typical size.

All polynomials are normalized by enforcing integer coefficients and no nontrivial integer content. Denote by $p=c_{0}+\cdots+c_{d} X^{d}$ the common univariate polynomial for given $N$ and call $d$ its degree. To reduce the size of the coefficients so as to display the polynomials, we remark that all coefficients remain integers under the substitution of $X$ with $X / 2^{2 N-3}$. The substituted polynomials are called $p^{\prime}=c_{0}^{\prime}+\cdots+c_{d}^{\prime} X^{d}$. See Table 1 and the sidebar below.

We also show on Table 2 the polynomials satisfied by $Q_{N-1}$ in any solution of $(3-11)-(3-12)$, for $2 \leq$ $N \leq 8$. They have degree 2 and illustrate the fact that $Q_{N-1}$ is the square root of a rational number.

\begin{tabular}{|c|rrrc|rccc|}
\hline & \multicolumn{4}{|c|}{ polynomial $p_{N}$} & \multicolumn{4}{|c|}{ polynomial $p_{N}^{\prime}$} \\
$N$ & $d$ & $s_{d}$ & $s_{0}$ & $\max _{i} s_{i}$ & $d$ & $s_{d}$ & $s_{0}$ & $\max _{i} s_{i}$ \\
\hline 2 & 2 & 1 & 1 & 1 & 2 & 1 & 1 & 1 \\
3 & 4 & 5 & 1 & 5 & 4 & 1 & 1 & 2 \\
4 & 8 & 13 & 3 & 13 & 8 & 1 & 3 & 4 \\
5 & 16 & 37 & 13 & 37 & 16 & 3 & 13 & 13 \\
6 & 32 & 87 & 29 & 87 & 32 & 1 & 29 & 29 \\
\hline
\end{tabular}

TABLE 1. Degrees and coefficient sizes of the polynomial $p$ satisfied by $Q_{0}$ for any solution of the system (3-11)-(3-12), and of the related polynomial $p^{\prime}$ obtained by the substitution $X \mapsto X / 2^{2 N-3}$. The notation $s_{i}$ denotes the number of digits of the (integer) coefficient of $X^{i}$ in the given polynomial.

\begin{tabular}{|cc|cc|cc|}
\hline 2 & $4 X^{2}-3$ & 4 & $64 X^{2}-35$ & 7 & $1024 X^{2}-429$ \\
3 & $8 X^{2}-5$ & 5 & $128 X^{2}-63$ & 8 & $16384 X^{2}-6435$ \\
& & 6 & $512 X^{2}-231$ & & \\
\hline
\end{tabular}

TABLE 2. Polynomials satisfied by $Q_{N-1}$ in any solution of (3-11)-(3-12), for $2 \leq N \leq 8$.

Finally, we turn to the refinement matrices for the construction proposed by Cohen, Daubechies and Vial in the case $N=2$.

$$
\begin{aligned}
p_{2}^{\prime}= & 2 X^{2}-2-1 \\
p_{3}^{\prime}= & 4 X^{4}-8 X^{3}-24 X^{2}-12+9 \\
p_{4}^{\prime}= & X^{8}-4 X^{7}-56 X^{6}-140 X^{5}+210 X^{4}+700 X^{3}-1400 X^{2}+500+625 \\
p_{5}^{\prime}= & 256 X^{16}-2048 X^{15}-122880 X^{14}-1162240 X^{13}+3672320 X^{12}+82199040 X^{11}-239052800 X^{10} \\
& -2639571200 X^{9}+21067452000 X^{8}-46192496000 X^{7}-73209920000 X^{6}+440535480000 X^{5} \\
& +344423450000 X^{4}-1907594500000 X^{3}-3529470000000 X^{2}-1029428750000+2251875390625 \\
p_{6}^{\prime}= & X^{32}-16 X^{31}-3968 X^{30}-127120 X^{29}+908488 X^{28}+99001616 X^{27}-206896256 X^{26}-45046412656 X^{25} \\
& +514227272860 X^{24}+9384914783664 X^{23}-326335992812928 X^{22}+3719423566862640 X^{21} \\
& -4725849211541640 X^{20}-321029601376721328 X^{19}+2420305333571518848 X^{18}+16398235495598877648 X^{17} \\
& -211208519547389641914 X^{16}-1033088836222729291824 X^{15}+9606191868945358307712 X^{14} \\
& +80272488735445037902416 X^{13}-74446118321296204796040 X^{12}-3691291866649887797453520 X^{11} \\
& -20403669167515311931757952 X^{10}-36966997633084650250167888 X^{9}+127608470131412725062780060 X^{8} \\
& +704247243896852021529183888 X^{7}-203778389811329721233161344 X^{6} \\
& -6143110504249885426575754992 X^{5}+3551450686163073382755632328 X^{4} \\
& +31306969279922401804098069360 X^{3}-61565775711706432446473031552 X^{2} \\
& +15639693023538327597289520112+61581291280182164914327485441
\end{aligned}
$$

Explicit form of the polynomials $p_{N}^{\prime}$, for $N=2, \ldots, 6$ (for definition, see text or caption of Table 1.) 
The filter coefficients $h_{k}$ of the Daubechies wavelets for the case $N=2$ are

$$
\begin{aligned}
h_{-1} & =\frac{1+\sqrt{3}}{4}, & h_{0} & =\frac{3+\sqrt{3}}{4}, \\
h_{1} & =\frac{3-\sqrt{3}}{4}, & h_{2} & =\frac{1-\sqrt{3}}{4} .
\end{aligned}
$$

The entries of the refinement matrices $H^{\text {edge }}$ and $G^{\text {edge }}$ are given in the sidebar below. For $k \geq N$ the entries are given by $H_{k, l}^{\text {edge }}=h_{l-2 k} / \sqrt{2}$ and $G_{k, l}^{\text {edge }}=$ $g_{l-2 k} / \sqrt{2}$.

\section{ACKNOWLEDGMENTS}

The calculations of the total degree Gröbner bases up to $N=34$ have been performed on the machines of the UMS MÉDICIS at the École Polytechnique in Palaiseau, France.
We thank Fabrizio Caruso, Philippe Dumas, Peter Maass, Zuhair Nashed, and Yang Wang for helpful comments and stimulating discussions.

\section{REFERENCES}

[Bradley et al. 1993] J. Bradley, C. Brislawn, and T. Hopper, "The FBI wavelet/scalar quantisation standard for gray-scale fingerprint image compression", Technical report, Los Alamos National Laboratory, 1993.

[Buchberger 1965] B. Buchberger, Ein Algorithmus zum Auffinden der Basiselemente des Restklassenringes nach einem nulldimensionalen Polynomideal, Ph.D. thesis, Philosophische Fakultät an der LeopoldFranzens-Universität, Innsbruck, Austria, 1965.

[Buchberger 1970] B. Buchberger, "Ein algorithmisches Kriterium für die Lösbarkeit eines algebraischen Gleichungssystems", Aequationes Mathematicae 4:3

$$
\begin{aligned}
& H_{0,0}^{\text {edge }}=\frac{\sqrt{2}(1137-119 \sqrt{3})}{2182} \quad H_{0,1}^{\text {edge }}=\frac{\sqrt{2} \sqrt{3969-2184 \sqrt{3}} \sqrt{242883-140092 \sqrt{3}}(16589+9619 \sqrt{3})}{14283372} \\
& H_{0,2}^{\text {edge }}=-\frac{\sqrt{2} \sqrt{3969-2184 \sqrt{3}}(123+85 \sqrt{3})}{13092} \\
& H_{1,0}^{\mathrm{edge}}=\frac{\sqrt{2} \sqrt{3969-2184 \sqrt{3}} \sqrt{242883-140092 \sqrt{3}}(32238331+17965009 \sqrt{3})}{501189240108} \\
& H_{1,1}^{\text {edge }}=\frac{\sqrt{2}(999+238 \sqrt{3})}{4364} \quad H_{1,2}^{\text {edge }}=\frac{\sqrt{2} \sqrt{242883-140092 \sqrt{3}}(2963297+1744500 \sqrt{3})}{153128396} \\
& H_{1,4}^{\text {edge }}=-\frac{\sqrt{2} \sqrt{242883-140092 \sqrt{3}}(897+445 \sqrt{3})}{280712}=-\frac{H_{1,4}^{\text {edge }}}{\sqrt{3}} \\
& G_{0,0}^{\text {edge }}=\frac{\sqrt{2826138238+1021826769 \sqrt{3}} \sqrt{3969-2184 \sqrt{3}}(6136686+2872499 \sqrt{3})}{12905163547593} \\
& G_{0,1}^{\text {edge }}=-\frac{\sqrt{2826138238+1021826769 \sqrt{3}} \sqrt{242883-140092 \sqrt{3}}(2102042+1389397 \sqrt{3})}{8603442365062} \\
& G_{0,2}^{\text {edge }}=\frac{\sqrt{2826138238+1021826769 \sqrt{3}}(80542-29121 \sqrt{3})}{7885831682} \\
& G_{1,0}^{\text {edge }}=\frac{\sqrt{35089} \sqrt{80542+29121 \sqrt{3}} \sqrt{3969-2184 \sqrt{3}}(605486-295683 \sqrt{3})}{8603442365062} \\
& G_{1,1}^{\text {edge }}=\frac{\sqrt{35089} \sqrt{80542+29121 \sqrt{3}} \sqrt{3969-2184 \sqrt{3}}(1147827+503061 \sqrt{3})}{17206884730124} \\
& G_{1,2}^{\text {edge }}=-\frac{\sqrt{35089} \sqrt{80542+29121 \sqrt{3}}(147657-115797 \sqrt{3})}{15771663364} \quad G_{1,4}^{\text {edge }}=\frac{\sqrt{35089} \sqrt{80542+29121 \sqrt{3}}}{140356}=-\frac{G_{1,3}^{\text {edge }}}{\sqrt{3}}
\end{aligned}
$$

Entries of the refinement matrices for the construction of Cohen, Daubechies and Vial in the case $N=2$. 
(1970), 271-272 and 374-383. English translation in [Buchberger and Winkler 1998].

[Buchberger and Winkler 1998] B. Buchberger and F. Winkler, Gröbner bases and applications, London Math. Soc. Lecture Notes Series 251, Cambridge University Press, Cambridge, 1998.

[Cohen and Daubechies 1993] A. Cohen and I. Daubechies, "Orthonormal bases of compactly supported wavelets, III: Better frequency resolution", SIAM J. Math. Anal. 24:2 (1993), 520-527.

[Cohen et al. 1992] A. Cohen, I. Daubechies, and J.-C. Feauveau, "Biorthogonal bases of compactly supported wavelets", Commun. Pure Appl. Math. 45:5 (1992), 485-560.

[Cohen et al. 1993a] A. Cohen, I. Daubechies, B. Jawerth, and P. Vial, "Multiresolution analysis, wavelets and fast algorithms on an interval", C. R. Acad. Sci. Paris, sér. 1 316:5 (1993), 417-421.

[Cohen et al. 1993b] A. Cohen, I. Daubechies, and P. Vial, "Wavelets on the interval and fast wavelet transforms", Appl. Comput. Harmon. Anal. 1:1 (1993), 5481.

[Cox et al. 1998] D. Cox, J. Little, and D. O'Shea, Using algebraic geometry, Springer-Verlag, New York, 1998.

[Dahmen and Schneider 1998] W. Dahmen and R. Schneider, "Wavelets with complementary boundary conditions - functions spaces on the cube", Results in Mathematics 34 (1998), 255-293.

[Dahmen et al. 1999] W. Dahmen, A. Kunoth, and K. Urban, "Biorthogonal spline wavelets on the interval - stability and momentum conditions", Appl. Comput. Harmon. Anal. 6:2 (1999), 132-196.

[Daubechies 1988] I. Daubechies, "Orthonormal bases of compactly supported wavelets", Commun. Pure Appl. Math. 41:7 (1988), 901-996.

[Daubechies 1992] I. Daubechies, Ten lectures on wavelets, CBMS-NSF Regional Conference Series in Applied Mathematics 61, SIAM, Philadelphia, 1992.

[Daubechies 1993] I. Daubechies, "Orthonormal bases of compactly supported wavelets, II: Variations on a theme", SIAM J. Math. Anal. 24:2 (1993), 499-519.

[DeVore et al. 1992] R. DeVore, B. Jawerth, and B. Lucier, "Image compression through wavelet transform coding", IEEE Trans. Inf. Theory 38:2/II (1992), 719-746.
[Faugère 1994] J. Faugère, Résolution des systèmes d'équations polynomiales, Ph.D. thesis, Université Paris VI - Pierre et Marie Curie, 1994.

[von zur Gathen and Gerhard 1999] J. von zur Gathen and J. Gerhard, Modern Computer Algebra, Cambridge University Press, Cambridge, 1999.

[Graham et al. 1994] R. Graham, D. Knuth, and O. Patashnik, Concrete Mathematics, 2nd ed., AddisonWesley, 1994.

[Jaffard 1992] S. Jaffard, "Wavelet methods for fast resolution of elliptic problems", SIAM J. Numer. Anal. 29:4 (1992), 965-986.

[Klappenecker 1997] A. Klappenecker, "On algebraic properties of selfreciprocal polynomials and of Daubechies filters of low order", pp. 80 in Proc. of 1997 IEEE Int. Symp. on Inform. Theory (Ulm, Germany, 1997), IEEE, New York, 1997.

[Mallat 1989] S. Mallat, "A theory for multiresolution signal decomposition: The wavelet representation", IEEE Trans. Pattern Anal. Mach. Intell. 11:7 (1989), 674-693.

[Meyer 1991] Y. Meyer, "Ondelettes sur l'intervalle", Rev. Mat. Iberoam. 7:2 (1991), 115-133.

[Paule and Schorn 1995] P. Paule and M. Schorn, "A Mathematica version of Zeilberger's algorithm for proving binomial coefficient identities", J. Symbolic Computation 20 (1995), 673-698.

[Petkovšek et al. 1996] M. Petkovšek, H. Wilf, and D. Zeilberger, $A=B$, A K Peters, 1996.

[Scherzer et al. 1998] O. Scherzer, A. Schoisswohl, and A. Kratochwil, "Wavelet Compression of 3D Ultrasound Data", Technical Report 7/1998, Industrial Mathematics Institute, Johannes Kepler University, Linz, 1998.

[Strang 1996] G. Strang, "Eigenvalues of Toeplitz matrices with $1 \times 2$ blocks", Z. Angew. Math. Mech. 76:2 (1996), 37-39.

[Williams and Amaratunga 1994] J. Williams and K. Amaratunga, "Introduction to wavelets in engineering", Int. J. Numer. Methods Eng. 37:14 (1994), 23652388 .

[Winkler 1996] F. Winkler, Polynomial Algorithms in Computer Algebra, RISC Texts and Monographs in Symbolic Computation, Springer, Vienna, 1996. 
Frédéric Chyzak, INRIA Rocquencourt, F-78153 Le Chesnay, France (Frederic.Chyzak@inria.fr)

Peter Paule, Research Institute for Symbolic Computation, Johannes Kepler University, A-4040 Linz, Austria (Peter.Paule@risc.uni-linz.ac.at)

Otmar Scherzer, Angewandte Mathematik, Universität Bayreuth, D-95440 Bayreuth, Germany (otmar.scherzer@uni-bayreuth.de)

Armin Schoisswohl, Kretztechnik AG, A-4871 Zipf, Austria (schoisswohl@kretz.co.at)

Burkhard Zimmermann, Research Institute for Symbolic Computation, Johannes Kepler University, A-4040 Linz, Austria (Burkhard.Zimmermann@risc.uni-linz.ac.at)

Received August 23, 1999; accepted in revised form May 15, 2000 